\title{
Percepción de docentes y orientadores de preparatorias públicas sobre sus prácticas tutoriales
}

\section{Perception of teachers and counselors of public high schools about their tutorial practices}

\author{
Edith Alcázar Carreño \\ edith.alcazar@uaem.mx \\ Enrique Vega Villanueva \\ evegavi@gmail.com
}

\author{
Doris Castellanos Simons \\ dcastellanos@uaem.mx \\ Rossana Pérez Andrade \\ rossanap@gmail.com
}

Universidad Autónoma del Estado de Morelos

\section{Resumen}

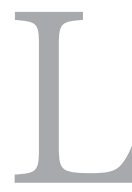

as tutorías forman parte de esfuerzos para apoyar el desarrollo integral de estudiantes en los centros educativos. El objetivo del estudio fue analizar las percepciones de tutores sobre sus prácticas tutoriales en tres escuelas preparatorias públicas de Cuernavaca. Participaron docentes $y$ orientadores $(\mathrm{N}=30)$, que fungieron como tutores en 1er año del ciclo escolar 2014- 2015. Los datos se obtuvieron mediante cuestionarios y grupos focales, comparándose las opiniones de los participantes sobre la implementación y práctica del modelo vigente. Los resultados destacan la insuficiente delimitación de funciones de docentes y orientadores, la falta de trabajo en equipo, la necesidad de capacitación en estrategias tutoriales y la percepción de baja eficacia por parte de los tutores respecto su labor tutorial.

Palabras clave: tutoría, docentes, orientadores educativos

\section{Summary}

he tutorials are part of efforts to support the integral development of students in schools. The objective of the study was to analyze the perceptions of tutors about their tutorial practices in three public high schools in Cuernavaca. In the study participated teachers and counselors $(\mathrm{N}=30)$, who served as tutors in the first year of the 2014-2015 school year. Data were obtained through questionnaires and focus groups, comparing the opinions of the participants on the implementation and practice of the current model. The results point out the insufficient delimitation of the functions of teachers and counselors, the lack of teamwork, the need for training in tutorial strategies and the perception of low effectiveness by the tutors regarding their tutorial work.

Key words: tutoring, teachers, educational advisors 


\section{Artículos Empíricos}

\section{Introducción}



n el contexto actual de cambio que atraviesa la educación en México, los programas tutoriales se han convertido en una estrategia importante para enfrentar los diversos retos educativos vinculados con la Reforma Integral de la Educación Media Superior (RIEMS), que no solo se enfoca a los aspectos académicos, sino al desarrollo integral del estudiante. La Asociación Nacional de Universidades e Instituciones de Educación Superior (ANUIES) destaca que, con una matrícula estudiantil que se eleva cada vez más, el requisito de una atención de calidad a toda la población estudiantil y el aprovechamiento de posibilidades de colaboración entre todos los vinculados de forma innovadora y dinámica se vuelve un imperativo (ANUIES, 2000).

La tutoría constituye un proceso de acompañamiento durante la formación de los estudiantes, por parte de profesores competentes; en este proceso el tutor es quien orienta, asesora y acompaña al estudiante durante su estancia en la institución, para guiarlo en su formación integral, estimulando en él la capacidad de hacerse responsable de su propio aprendizaje y de su formación (ANUIES, 2000). Siguiendo la propuesta de la ANUIES para formalizar las estrategias que apoyen al desarrollo integral del estudiante, se implementa en la Universidad Autónoma del Estado de Morelos (UAEM) el modelo tutorial vigente desde 2015, que la concibe como "...una orientación sistemática $\mathrm{y}$ formal que se le proporciona a un estudiante para apoyar su avance académico, personal $\mathrm{y}$ profesional conforme a sus necesidades $\mathrm{y}$ requerimientos particulares, dentro del contexto académico" (Plan Institucional de Tutorías (PIT), 2015, pp.12-13).

El objetivo principal del PIT es contribuir al fortalecimiento de habilidades intelectuales, actitudes y valores del estudiante, durante su trayectoria escolar, mediante estrategias y acciones para una atención de carácter personalizado, en forma grupal o individualizada en sus diferentes tipos y modalidades, en los Niveles Medio Superior, Superior y Posgrado. Establece las diversas figuras del tutor, relacionadas con las funciones de: asesoría, consejería, orientación, dirección de tesis, acompañamiento en contexto, acompañamiento académico. Por otra parte, según el Plan Institucional de Desarrollo Educativo 2012-2018 (PIDE) de la UAEM, las funciones establecidas vinculan igualmente el trabajo de las tutorías con las funciones del Orientador Educativo en las áreas: a) Personal, b) Escolar, c) Vocacional, d) Familiar, e) Social, f) Institucional. Así, en los documentos programáticos de la institución, la tutoría y la orientación educativa conforman una labor compartida entre profesores y orientadores.

De acuerdo con lo anteriormente expuesto, resulta fácil observar las relaciones entre las acciones definidas para el profesor tutor y el orientador educativo, y el hecho que existen marcadas coincidencia (e incluso solapamiento) entre sus respectivas funciones, en las tareas relacionadas con el acompañamiento y apoyo al desarrollo integral del estudiante. En este sentido, Álvarez y Bizquerra (2008) enfatizan que la atención al alumno requiere de la implicación y participación conjunta de todos los agentes educativos para contribuir al desarrollo global de todos los estudiantes. Aunque se reconoce 
que lo idóneo es trabajar en la integralidad de la persona, con un trabajo coordinado entre el orientador y tutor, para garantizar que estudiante se sienta acompañado en su proceso académico (Álvarez \& Rodríguez, 2000, Asensi, 2010), pueden generarse contradicciones entre ambos así como la percepción de ambigüedades en la definición de sus respectivos roles.

Algunas de las investigaciones en torno a la implementación de las prácticas tutoriales (García, Cuevas, Vales \& Cruz, 2012; Lázaro \& Asensi, 1990), han evidenciado la existencia de barreras y facilitadores del trabajo tutorial que realizan conjuntamente docentes y orientadores, así como las percepciones que ambos tienen de sus funciones, de la forma de llevarlas a la práctica y de su contribución al desarrollo de los estudiantes.

Así por ejemplo, Rubio y Martínez (2012), en un estudio realizado en una escuela preparatoria pública de San Luis Potosí, que a partir de 2006 incorporó curricularmente la orientación y la tutoría, examinaron la percepción de tutores, orientadores y estudiantes sobre las condiciones laborales y la carga de trabajo, así como sobre la forma en que tutores y orientadores se vinculaban laboralmente para atender a la población estudiantil. Encontraron que los alumnos valoraban la atención como inadecuada, los docentes percibían la carga laboral demasiado elevada ya que no se podía atender a la diversidad, y los orientadores registraban que sus compañeros docentes-tutores desconocían las funciones tutoriales. En general, la percepción sobre la implementación de las tutorías fue negativa por parte de orientadores y docentestutores, así como la valoración del conocimiento de las respectivas funciones.
En contraste, en un estudio realizado por Fuentes y Prieto (2013) en una preparatoria pública de Guadalajara, se analizaron mediante un cuestionario las percepciones de orientadores y tutores sobre la colaboración para implementar sus funciones. Se destacó que los orientadores escolares afrontan el reto del trabajo en equipo desde una perspectiva colaborativa, sistémica e interdisciplinar, que involucra a los tutores docentes; perciben que más de un $50 \%$ de las tareas del orientador educativo implican colaboración con el trabajo tutorial de los docentes, en particular en los rubros académico y profesional (66,67\%). Mientras, el 33\% de los tutores percibe que las tutorías ofrecen apoyo al desarrollo personal, además del académico y que requieren por ello del trabajo en equipo. Se concluye entonces la necesidad del trabajo colegiado entre orientadores y docentes-tutores, y de una percepción positiva de éste.

Asimismo, en la investigación de Alcázar (2012) en una preparatoria publica de Morelos, dirigida a evaluar la efectividad de una estrategia de tutoría entre pares para apoyo a alumnos con riesgo de deserción escolar, la participación conjunta de orientadores educativos (quienes aportaron información escolar y académica de alumnos en riesgo de deserción) y de los docentes tutores (quienes brindaron la información acerca los procesos de aprendizaje y motivacionales de los estudiantes) resaltó la importancia del trabajo en equipo para lograr la caracterización del estudiantado como base de las tutorías.

Según Asensi (2010), la concepción de las tutorías como una labor participativa ha ido afianzándose cada vez más. El trabajo en equipos conformados por docentes tutores, 


\section{Artículos Empíricos}

orientadores educativos y la administración del centro escolar, eleva la calidad de las acciones tutoriales y el esfuerzo para el logro de las metas correspondientes.

No obstante, la eficacia de la labor colegiada depende en gran medida de las percepciones $\mathrm{y}$ actitudes de sus responsables- docentes y orientadores que fungen como tutores- sobre sus respectivas funciones y tareas, así como sobre el apoyo recíproco y la necesidad de participación en cada uno en las mismas. Las prácticas tutoriales (colegiadas o no) exigen comprender dicha colaboración y valorar positivamente del papel de los diferentes tutores en estos procesos. El conocimiento y la delimitación de estas responsabilidades parecen esenciales $y$ no siempre se logra. Por ello, resulta relevante obtener información acerca de las percepciones que tienen ambos tipos de actores sobre las tutorías y su potencial en la atención al desarrollo integral de los alumnos, lo cual a menudo no se ha tomado en cuenta.

Precisamente el presente estudio estuvo dirigido a comparar las percepciones que tienen docentes y orientadores implicados en las tutorías, sobre las prácticas que se implementan en tres escuelas preparatorias de la UAEM, y analizar su relación con la manera en que se comprenden las funciones asignadas en el modelo tutorial vigente al docente-tutor y al orientador.

\section{Método}

Se reporta un estudio descriptivo con enfoque mixto. La primera etapa, cualitativa, estuvo dirigida a obtener información sobre el conocimiento sobre el programa tutorial vigente en la institución y sobre las funciones tutoriales a partir de las perspectivas de orientadores y docentes; en la segunda etapa, cuantitativa, se examinaron las valoraciones sobre ambos tipos de tutores sobre la eficacia de las tutorías.

\section{Participantes:}

El estudio se realizó en 3 escuelas públicas de nivel medio superior pertenecientes a la UAEM. Dos de las escuelas (codificadas como P1 y P2) eran preparatorias generales, matutina y vespertina respectivamente. La tercera escuela (P3) era una preparatoria bivalente. En la primera etapa (grupos focales) se incorporaron en un primer grupo focal, 5 orientadores educativos que en el momento de la investigación no fungían como tutores y en un segundo grupo focal, 7 tutores docentes (TD) y 2 tutores orientadores (TO), para un total de 14 participantes. En una segunda etapa, participaron 30 tutores (23 TD y 7 TO) para evaluar la percepción de las tutorías. Además de la disposición a integrarse en el proyecto, la selección de los participantes se realizó sobre los siguientes criterios: a) Participar en el programa de tutorías o haber trabajado con los estudiantes en cualquiera de las alternativas tutoriales, b) Conocer el PITUAEM. En conjunto, el $76.7 \%$ pertenecían a las dos preparatorias generales y el 23,3\% la bivalente, con edad promedio de 44.5 años.

Grupos focales:

Se desarrollaron 2 grupos focales con orientadores educativos y con tutores (docentes y orientadores), con el objetivo de examinar el conocimiento que tenían sobre: el modelo tutorial de la institución, funciones de las tutorías, contribución de las prácticas a los fines con que persiguen. 
Materiales:

Cuestionario de percepción de la acción tutorial $(C P A T)$. Se trata de un instrumento adaptado al Nivel Medio Superior a partir del cuestionario utilizado por la ANUIES con el propósito evaluar la percepción de las estrategias tutorales implementadas en la escuela y que consta de sendas versiones para tutores y estudiantes (se reporta la primera). Está compuesto por 47 preguntas en escala Likert (4 valores) y 3 preguntas abiertas. Comprende dos dimensiones: 1) Percepción de las prácticas tutoriales (PT), con cinco subdimensiones y 38 ítems; 2) Percepción las características y del desempeño de los tutores (DT) con dos subdimensiones y 9 ítems. El cuestionario, sometido también a valoración por expertos, demostró adecuada confiabilidad con coeficientes Alfa de Cronbach de 0.98 y 0.93 en sus dimensiones 1 y 2 , y coeficientes entre 0.87 y 0.95 para las 7 subdimensiones.

\section{Procedimiento}

Después de gestionar con los directivos el consentimiento de las escuelas para su participación en la investigación, se realizó una convocatoria a los orientadores y a los docentes tutores, se les explicó el proyecto de investigación y se les invitó a participar. Con aquellos que accedieron, se realizaron sucesivamente 2 grupos focales en las instalaciones de las escuelas. La primera etapa cerró con el análisis de los datos obtenidos en estos y el pilotaje del cuestionario adaptado en una escuela preparatoria de la UAEM que no participó en la investigación. En una segunda etapa de evaluación de la implementación de las prácticas tutoriales se aplicaron los cuestionarios en varias sesiones, en función de la disponibilidad de los participantes, en salones de las preparatorias.
A nivel cualitativo, se procesaron los datos provenientes de los grupos focales y, se realizó un análisis de contenido de las respuestas de las participantes previamente transcritas, destacándose las principales categorías emergentes y sus relaciones. A nivel cuantitativo, se procesaron las preguntas cerradas del CPAT con ayuda del paquete estadístico SPSS (Versión 23), y a partir de pruebas de estadística descriptiva e inferencial paramétricas puesto que el análisis de normalidad resultó positivo para sus variables. Se trabajó con un nivel de significación de 0.05.

\section{Resultados}

Primer grupo focal con orientadores - no tutores.

El primer grupo focal se desarrolló con orientadores educativos que no fungían como tutores para examinar la visión de las funciones del tutor y prácticas tutoriales, desde su propia área y perspectiva. Por razones éticas, se refieren los participantes identificados por un código que representa sus iniciales, y su escuela preparatoria $(\mathrm{P} 1, \mathrm{P} 2)$

A partir del análisis de las aportaciones de los orientadores en el grupo focal, se evidenció que funciones básicas que reconocen se relacionan fundamentalmente con el trabajo integral con los estudiantes por medio de tareas como:

a) Desarrollo de programas frente a grupo basados en competencias,

b) Acompañamiento y tutoría al estudiante,

c) Exposiciones profesiográficas,

d) Talleres Psicopedagógicos sobre 


\section{Artículos Empíricos}

Prevención de Adicciones y Manejo de Emociones.

Según los participantes, su función se relaciona con el desarrollo de los estudiantes a menos en los siguientes aspectos:

- Apoyarlos en el diseño de un proyecto de vida coherente con sus características.

- Favorecer sus procesos de maduración vocacional y profesional.

- Apoyar en el desarrollo integral de la personalidad.

- Contribuir a la coordinación y vinculación de todos los profesores/as implicados en los procesos de enseñanzaaprendizaje.

Los participantes destacan que el trabajo tutorial presenta grandes desafíos, relacionados con los principales problemas detectados por ellos como el bajo aprovechamiento, una matrícula elevada, altas tasas de reprobación, y deficiente práctica de valores. Las limitaciones asociadas con la infraestructura, así como con los tiempos destinados a la tutoría, el número elevado de estudiantes que definen sobre todo, la exigencia del trabajo grupal no personalizado, son aspectos que se destacan. Así, por ejemplo, una de las orientadoras, señala:

"Se da una atención a medias, ya que existe la falta de tiempo asignado para los momentos de tutorías. Probablemente no se entienda toda la diversidad de estudiantes; no, sobre todo en la modalidad en la que estamos trabajando las tutorías, que es grupal...creo que debe haber momentos establecidos para casos particulares, y no se tiene un lugar específico para atención personalizada". (AL-P1)

Por otro lado, también se evidenció la falta de diferenciación entre las actividades de orientación y la tutoría:

“...La orientación educativa la confunden (los alumnos) con la tutoría, porque creo que no hay mucha diferencia en los temas. Entonces dicen, voy con mi tutora, y van con la orientadora, y eso crea también barreras para trabajar con toda la población, ya que no asisten con su tutor realmente". (IA-P1).

En síntesis, las barreras que debilitan o dificultan el trabajo en su área académica, son, a juicio de los participantes:

- Existencia de una planta docente con falta de compromiso hacia la tutoría y centrada en sus propias actividades académicas.

- Falta de asistencia y participación de los alumnos en las actividades de tutoría.

- Falta de trabajo en equipo en la comunidad educativa (docentes y administración de la escuela trabajando de manera aislada).

- Infraestructura inadecuada para dar atención tutorial.

- No existen tutores de tiempo completo.

Segundo grupo focal con docentes-tutores y orientadores educativos-tutores

La información recolectada permitió examinar con mayor detalle la percepción de las prácticas tutoriales desde la perspectiva de 
las personas involucradas como tutores, quienes desarrollan las acciones y estrategias de tutorías en las escuelas.

La falta de capacitación y actualización de los docentes en cuanto a las prácticas y estrategias tutoriales y de manejo de grupo se señaló como cuestión esencial:

“...yo tengo otra formación, siempre digo esto porque, o sea, en dónde me voy a respaldar yo para poder apoyar, y no solucionar su problema, sino para apoyar y darle un acompañamiento, pero que el joven se sienta integrado, porque él se está sintiendo excluido, sí me hacen falta herramientas para poder ayudar, para darle seguimiento al joven" (Docente-Tutora, AA-P2).

Los aspectos más importantes en los comentarios de los tutores apuntan también a las preocupaciones respecto a estrategias de atención a la diversidad, tanto en profesores como en orientadores. Existe conciencia a la diversidad del estudiantado, sin embargo, por la cantidad en cada aula ha sido complicada la atención, dicen los tutores, se queda a medias. La principal barrera destacada en la práctica tutorial tiene que ver entonces con el tiempo insuficiente brindado a las tutorías, y la cantidad de estudiantes (tener 50 en el aula para atender), así como el hecho de que los estudiantes no le den importancia a las tutorías.

Se enfatiza, entonces, la necesidad de compartir el trabajo y el apoyo entre ambos tipos de tutores: "Creo que son diversas las acciones que realizamos como equipos de tutores (...) socializamos los casos, (...) nos apoyamos con información enriquecimiento nuestra tarea como tutor..." (Orientadora, AL, P1). Sin embargo, algunos reconocen que no se comparten los mismos temas en tutoría: "Aunque no compartimos los mismos temas en tutorías, compartimos experiencias con las estrategias de integración de grupo, en el desarrollo académico..." (Docente, BP, P3). En general, en el grupo focal las intervenciones se centraron en la necesidad de compromiso con un equipo, y evidenciaron la percepción de iniciativa y disposición de los tutores, aunque se manifiesta la necesidad de los docentes de ser capacitados en estrategias concretas.

La aplicación del cuestionario (CPAT) permitió evidenciar con más detalles las valoraciones de los tutores docentes y orientadores respecto a las prácticas tutorales implementadas.

Opiniones de tutores (docentes y orientadores) sobre las prácticas tutoriales. Resultados del CPAT

La Tabla 1 presenta resultados generales que permiten comparar en el grupo de tutores las dos dimensiones estudiadas. Los valores han sido llevados a puntuaciones centiles para hacerlos comparables. No se observan diferencias marcadas entre las dos dimensiones aunque sí marcada dispersión de los puntajes. Para el grupo general de tutores, independientemente de su perfil, el aspecto menos valorado dentro de las funciones es el relacionado con la atención a la permanencia e integración académica del estudiante. 


\section{Artículos Empíricos}

Tabla 1

Estadísticas descriptivas para las dimensiones y subdimensiones del Cuestionario de Percepción de la Acción Tutorial (tutores en general, $N=30$ ).

Dimensiones y Subdimensiones

\begin{tabular}{|c|c|c|c|c|}
\hline & Media & $\begin{array}{l}\text { Desviación } \\
\text { estándar }\end{array}$ & Máximo & Mínimo \\
\hline Percepción de la acción tutorial & 52.68 & 34.013 & 99.1 & 2.6 \\
\hline 1 Integración y permanencia del estudiante & 49.30 & 28.765 & 95.8 & 8.3 \\
\hline 2 Procesos de aprendizaje y escolares & 54.66 & 38.242 & 100.0 & .0 \\
\hline 3 Desarrollo vocacional y profesional & 53.55 & 34.86 & 100.0 & .0 \\
\hline 4 Desarrollo personal y social & 51.66 & 36.14 & 100.0 & .0 \\
\hline 5 Desarrollo de las prácticas Inclusivas & 55.42 & 37.05 & 100.0 & .0 \\
\hline Percepción y desempeño del tutor & 53.89 & 40.09 & 100.0 & .0 \\
\hline 1 Compromiso con la actividad tutorial (actitud) & 53.10 & 40.81 & 100.0 & .0 \\
\hline $\begin{array}{l}2 \text { Capacidad para la acción tutorial (conocimientos, } \\
\text { habilidades y competencias) }\end{array}$ & 55.17 & 40.17 & 100.0 & .0 \\
\hline
\end{tabular}


Tabla 2

Comparación entre las escuelas participantes en subdimensiones del CPAT $(N=30)$

\begin{tabular}{|c|c|c|c|c|c|c|}
\hline \multirow{3}{*}{$\begin{array}{l}\text { Subdimensiones y } \\
\text { dimensiones }\end{array}$} & \multicolumn{6}{|c|}{ Escuelas Preparatorias } \\
\hline & \multicolumn{2}{|l|}{$\mathrm{P} 1$} & \multicolumn{2}{|l|}{$\mathrm{P} 2$} & \multicolumn{2}{|l|}{ P3 } \\
\hline & Media & Desviación & Media & Desviación & Media & Desviación \\
\hline $\begin{array}{l}\text { Integración y } \\
\text { permanencia del } \\
\text { estudiante. }\end{array}$ & 73.60 & 17.45 & 27.61 & 14.41 & 24.16 & 7.44 \\
\hline $\begin{array}{l}\text { Procesos de aprendizaje } \\
\text { y escolares. }\end{array}$ & 90.65 & 11.49 & 22.50 & 9.37 & 16.02 & 12.11 \\
\hline $\begin{array}{l}\text { Desarrollo vocacional y } \\
\text { profesional. }\end{array}$ & 85.33 & 11.04 & 29.16 & 12.83 & 14.66 & 15.19 \\
\hline $\begin{array}{l}\text { Desarrollo personal y } \\
\text { social. }\end{array}$ & 84.25 & 14.10 & 23.26 & 14.90 & 13.90 & 16.32 \\
\hline $\begin{array}{l}\text { Desarrollo de las } \\
\text { prácticas Inclusivas. }\end{array}$ & 89.64 & 12.67 & 24.97 & 10.96 & 17.76 & 15.12 \\
\hline $\begin{array}{l}\text { Total Dimensión } \\
\text { Percepción de la acción } \\
\text { tutorial. }\end{array}$ & 84.28 & 11.08 & 25.22 & 10.33 & 17.26 & 11.84 \\
\hline $\begin{array}{l}\text { Compromiso con la } \\
\text { actividad tutorial } \\
\text { (actitud). }\end{array}$ & 90.21 & 15.70 & 22.50 & 16.30 & 5.32 & 7.28 \\
\hline $\begin{array}{l}\text { Capacidad para la acción } \\
\text { tutorial (conocimientos, } \\
\text { habilidades y } \\
\text { competencias). }\end{array}$ & 89.63 & 23.18 & 23.58 & 13.83 & 17.76 & 20.16 \\
\hline $\begin{array}{l}\text { Total Dimensión } \\
\text { Características y } \\
\text { desempeño del tutor. }\end{array}$ & 90.00 & 17.86 & 22.92 & 14.08 & 10.00 & 11.99 \\
\hline
\end{tabular}


Se encontraron diferencias resultaron estadísticamente significativas entre las escuelas en todas las subdimensiones y dimensiones al calcularse un ANOVA para cada dimensión como variable dependiente con la escuela como factor con tres valores (P1, P2, P3). El análisis post-hoc utilizando la Prueba de Tukey arrojó diferencias significativas entre las tres escuelas en subdimensión 2 (Contribución a la Integración y Permanencia del Estudiante), con puntajes superiores para la P1 en comparación con P2 y P3. En las restantes subdimensiones la P1 puntuó significativamente más alto que las restantes escuelas, pero en ningún caso se encontraron diferencias significativas entre P2 y P3. En síntesis, los tutores de estas dos últimas escuelas no se diferenciaron significativamente en sus percepciones sobre sus prácticas tutoriales y sus propias competencias y actitudes para su desempeño.

Finalmente la Tabla 3 permite observar las diferencias entre docentes y orientadores, respecto a los principales ejes de contribución de su labor tutorial.

Tabla 3

Diferencia de medias entre Docentes-Tutores ( $n=23)$ y Orientadores-Tutores $(n=7)$ en cada una de las dimensiones y subdimensiones del CPAT.

\begin{tabular}{lllll}
\hline Subdimensiones y dimensiones & \multicolumn{2}{l}{ Perfil del tutor } \\
& Docente & \multicolumn{3}{c}{ Orientador } \\
\hline Subdimensiones y dimensiones & Media & Desviación & Media & Desviación \\
\cline { 2 - 5 } Integración y permanencia del estudiante. & 36.25 & 25.94 & 75.41 & 10.47 \\
Procesos de aprendizaje y escolares. & 37.00 & 34.08 & 89.99 & 13.42 \\
Desarrollo vocacional y profesional. & 36.99 & 30.85 & 86.67 & 8.89 \\
Desarrollo personal y social. & 33.89 & 30.32 & 87.21 & 12.70 \\
Desarrollo de las prácticas Inclusivas. & 37.95 & 32.09 & 90.38 & 14.61 \\
Total Percepción de la acción tutoral. & 36.11 & 29.19 & 85.82 & 9.94 \\
\hline Compromiso con la actividad tutorial (actitud). & 35.66 & 37.60 & 87.99 & 18.80 \\
Capacidad para la acción tutorial (conocimientos, & 38.32 & 34.67 & 88.89 & 27.71 \\
habilidades y competencias). & & & & \\
Total Características y desempeño del tutor. & 36.66 & 36.02 & 88.34 & 21.48 \\
\hline
\end{tabular}


En general en todos los rubros evaluados, como se observa en la Tabla 3, los orientadores tienden a evaluar mucho mejor la contribución de sus prácticas tutoriales a las diferentes áreas del desarrollo de los estudiantes. Sin embargo, las diferencias de medias no resultaron significativas (prueba $t$ para muestras independientes).

\section{Discusión}

El presente estudio estuvo centrado en examinar las percepciones que tienen los tutores (docentes y orientadores) sobre sus prácticas tutoriales, desde las perspectivas de su perfil y sus funciones. La investigación se desarrolló en tres preparatorias con condiciones diferentes para la implementación de estrategias tutoriales, fundamentalmente con relación con el número de tutores, mucho mayor en la P1. Desde un enfoque mixto se accedió e integró información proveniente de sus valoraciones brindadas en grupos focales y en un cuestionario, que abarcaron dos grandes dimensiones: la autovaloración de la contribución de sus prácticas a los objetivos del modelo tutorial vigente en la institución (centrado en la integración de los estudiantes, en su desarrollo personal, social y académico), y la de sus propias competencias y actitudes para el ejercicio de esta labor.

En línea con los resultados encontrados por Asenci (2010) y Rubio y Martínez (2012) se destaca en este estudio que las funciones tutoriales se comparten por profesores y tutores, y que no siempre éstos distinguen las mismas, así como tampoco son conscientes a menudo que la colaboración y el enfoque participativo son decisivos para su eficacia. En este sentido, la experiencia concreta del trabajo en equipo aporta claridad a la comprensión de los roles, como se evidenció en este estudio, en aquellos que se están desempeñando como tutores y que lo valoran. En esta investigación, los orientadores que no habían fungido previamente como tutores mostraron, en el primer grupo focal, menor sensibilización respecto a la necesidad del trabajo colegiado, centraron la orientación fundamentalmente en los aspectos personales, vocacionales y profesionales. Aquellos docentes y orientadores que han habían tenido con antelación experiencia en las tutorías coincidieron en destacar la importancia de la colaboración y ayuda recíproca para enfrentar los retos de la tarea que, desde el propio modelo vigente en la UAEM incluye funciones tan diversas como; asesoría, consejería, orientación, entre otras, que pueden ser desarrolladas por una u otra figura. Por otra parte, acorde con lo encontrado por Ruiz y Martínez (2012), los orientadores opinaron que sus colegas profesores tienen poco conocimiento sobre las funciones tutoriales. Un hallazgo interesante resultó ser el que los orientadores tengan una autovaloración más elevada de la eficacia de su labor tutorial en diferentes rubros.

En este sentido, la formación y experiencia de los orientadores responde, más que la de los docentes, a las exigencias de la actividad tutorial, lo que podría explicar que los primeros perciben más positivamente su contribución a los objetivos tutoriales. El propio modelo tutorial vigente en la UAEM no facilita la clara delimitación entre las funciones tutoriales que deben realizar los docentes, y las que se realizan desde la orientación educativa. Adicionalmente, los docentes han resaltado la necesidad de la capacitación en estrategias tutoriales, y probablemente, sus puntajes bastante bajos en el CPAT reflejen a su vez la inseguridad en las 


\section{Artículos Empíricos}

propias competencias para realizar estas tareas, además de la visión de la misma como una carga excesiva.

Por otro lado, tanto tutores y orientadores educativos coinciden en las principales problemáticas relacionadas la adecuada implementación de las prácticas: la falta de tiempo de los tutores, sobrecarga de funciones, insuficiente cantidad de tutores y de sesiones de tutoría, la inadecuada organización y planificación de la misma, la vinculación con el resto de los docentes, en algunos casos, la falta compromiso y trabajo colaborativo entre la comunidad educativa. Acorde con Rubio y Martínez (2012) los tutores en general perciben poca motivación de parte de los estudiantes en las tutorías.

\section{Conclusión}

En síntesis, el estudio permitió destacar las expectativas y necesidades de los tutores

\section{Referencias}

Alcázar, E. (2012). La tutoría entre pares para la atención educativa a los alumnos en riesgo de deserción escolar en la Preparatoria (Tesis de grado de Maestra en Psicología). Facultad de Psicología. Universidad Autónoma del Estado de Morelos.

Álvarez, M. \& Rodríguez, S. (2000). Cambios socio-educativos $y$ orientación en el siglo XXI: nuevas estructuras, roles $y$ funciones. En: XII Congreso Nacional e y orientadores educativos, que deben ser de utilidad para repensar el futuro de la acción tutorial, de los propios modelos de tutorías de las instituciones, y la formación de los agentes educativos que la brindan. Algunas de las necesidades tienen que ver con la mejora de la capacidad de organización para abordar la acción tutorial; otras, con la capacitación de los tutores y el análisis racional de las demandas a cada una de las figuras que implementan la mismas; conllevan a la reflexión profunda sobre la relación entre profesores y tutores en cuanto a su trabajo conjunto. De acuerdo con los resultados obtenidos, la orientación educativa parece estar cubriendo la mayoría de las funciones tutoriales, así como el trabajo de los docentes en este renglón. La definición de los límites de cada uno, y del tipo de aporte particular que hacen a la acción tutorial es indispensable, y requiere también de una cultura del trabajo cooperativo en las escuelas que aún no se evidencia plenamente.

Iberoamericano de Pedagogía. Madrid: Sociedad Española de Pedagogía.

Álvarez, M. \& Bisquerra, R. (coord.). (2008). Manual de orientación y tutoría. Barcelona: Wolters Kluwer.

ANUIES (2000). Programas Institucionales de Tutoría. Una propuesta de la ANUIES para su organización y funcionamiento en las instituciones de educación superior. México: 
Colección Biblioteca de la Educación Superior. Serie Investigaciones, ANUIES.

Asensi, J. (2010). Notas sobre un cuento mítico sobre tutoría. Tendencias Pedagógicas, 15, 171-183.

García, R. I., Cuevas, O., Vales, J. J. \& Cruz, I. (2012). Impacto del Programa de Tutoría en el desempeño académico de los alumnos del Instituto Tecnológico de Sonora. Revista Electrónica de Investigación Educativa, 14 (1), 106-121. Recuperado de http://redie.uabc. $\mathrm{mx} /$ vol14no1/contenido-garciaetal.html
Secretaría de Educación Pública (SEP) (2009). Metodología para el Desarrollo de la Acción Tutorial para el Bachillerato General. México: SEP.

UAEM (2013a). Plan Institucional de Desarrollo Educativo 2012-2018. México: Universidad Autónoma del Estado de Morelos.

UAEM (2013b). Plan Institucional de Tutorías 2015. México: Universidad Autónoma del Estado de Morelos. 